\title{
An Analysis of the Post-Fault Behavior of Robotic Manipulators
}

\author{
M. Goel, A. A. Maciejewski, and V. Balakrishnan \\ Purdue University \\ School of Electrical and Computer Engineering \\ 1285 Electrical Engineering Building \\ West Lafayette, Indiana 47907-1285
}

\begin{abstract}
Operations in hazardous or remote environments are invariably performed by robots. The hostile nature of the environments, however, increase the likelihood of failures for robots used in such applications. The difficulty and delay in the detection and consequent correction of these faults makes the post-fault performance of the robots particularly important. This work investigates the behavior of robots experiencing undetected locked-joint failures in a general class of tasks characterized by point-to-point motion. The robot is considered to have "converged" to a task position and orientation if all its joints come to rest when the end-effector is at that position. It is seen that the post-fault behavior may be classified into three categories: 1 ) The robot converges to the task position; 2) the robot converges to a position other than the task position; or 3) the robot does not converge, but keeps moving forever. The specific conditions for convergence are identified, and the different behaviors illustrated with examples of simple planar manipulators.
\end{abstract}

\section{INTRODUCTION}

It is well-known that robotic manipulators fail quite frequently under normal industrial operation [1]. The situation is exacerbated when robots are required to operate in remote or hazardous environments. The very nature of such environments increases the likelihood of a failure. Since, immediate human intervention for repair or recovery is precluded, the post-fault performance of the manipulator assumes great importance.

There are many ways in which a manipulator can fail [2], and these are typically classified into different failure modes. The one considered in this work is the commonly used locked-joint model, where the affected joint's velocity is identically zero; this may be due to "locking up" caused by the failure itself, or where failsafe brakes have been applied. Such a failure may have catastrophic consequences, or, at the very least, significantly degrade the system performance. Therefore, the post-failure performance must be addressed in the overall design.

This work was supported by Sandia National Laboratories under contract number AL-3011.
One approach towards dealing with failures is to build fault-tolerant manipulators, where the damaged system can be operated with minimal performance degradation. Fecilure tolerance can be achieved in different ways; a popular approach is the incorporation of redundancy into the design. This can be in the form of duplicated critical components such as joint actuators $[7,8]$, or intelligent utilization of kinematic redundancy $[3,4,5,6]$. These techniques are frequently coupled with fault-detection and recovery mechanisms $[9,10,11]$.

Though there has been considerable work in the area of back-up systems and failure-detection, there remain significant questions about the post-fault behavior of robotic systems that do not incorporate these fail-safe mechanisms. Even in systems that incorporate these schemes, multiple failures can overwhelm back-up hardware, and fault-detection is rarely instantaneous. These issues are even more critical in teleoperated systems, where the operator may become disoriented by the erroneous motion of the arm by the time a corrective measure is taken [12]. These problems can be be addressed by eliminating the dependency on faultdetection and designing a control scheme that ensures acceptable performance even in the presence of faults.

In this work the behavior of robotic manipulators performing point-to-point motion tasks with undetected locked-joint failures is explored. In particular, we study convergence issues such as whether the manipulator comes to rest, and if so, what is the terminal position and orientation ${ }^{1}$ of the end-effector. Conditions under which the manipulator converges are explicitly defined and the anomalies in behavior due to the faults explained and illustrated with examples. The analysis also discusses the effect of faults on the manipulator workspace and presents j.nformation vital to workspace layout.

\footnotetext{
${ }^{1}$ We will henceforth use the term "position" to mean any combination of position and/or orientation variables.
} 


\section{Analysis of Convergence Behavior}

The position and orientation of the end-effector of a manipulator can be expressed in terms of its joint variables by the kinematic equation

$$
\mathrm{x}=f(\mathbf{q}),
$$

where $\mathrm{x} \in \mathbb{R}^{m}$ is the position of the end-effector, $\mathbf{q} \in \mathbb{R}^{n}$ is the vector of joint variables, and $m$ and $n$ the dimensions of the task-space and joint-space respectively. Manipulators that have more degrees-offreedom (DOF) than required for a task, i.e. $n>m$, are said to be redundant. The end-effector velocity is expressed in terms of the joint rates as

$$
\dot{\mathrm{x}}=J \dot{\mathbf{q}}
$$

where $J \in \mathbb{R}^{m \times n}$ is the manipulator Jacobian, $\dot{\mathrm{x}}$ is the end-effector velocity vector, and $\dot{\mathbf{q}}$ is the joint velocity vector.

If perfect servo-control of the joints is assumed, then in a healthy manipulator the actual joint velocities $\dot{\mathbf{q}}_{a}$ equal the commanded velocities $\dot{\mathbf{q}}_{c}$. However, in the event of a locked-joint failure of the $i$ th joint, the corresponding element of $\dot{\mathbf{q}}_{a}$ is identically zero. Then, the actual end-effector velocity is given by

$$
\dot{\mathbf{x}}_{a}={ }^{i} J \dot{\mathbf{q}}_{c},
$$

where ${ }^{i} J$ is the post-failure Jacobian, given by

$$
{ }^{i} J=\left[\begin{array}{lllllll}
\mathbf{j}_{1} & \cdots & \mathbf{j}_{i-1} & \mathbf{0} & \mathbf{j}_{i+1} & \cdots & \mathbf{j}_{n}
\end{array}\right] .
$$

It is assumed that the joint position sensors are still operational.

A common method for generating $\dot{q}$ is the inversekinematic scheme

$$
\dot{\mathrm{q}}=G \dot{\mathrm{x}}
$$

where $G$ is a generalized inverse of $J$ satisfying the Penrose condition $J G J=J$. A frequently encountered generalized inverse is the pseudoinverse $J^{+}$, which yields the least-squares minimum norm solution. For fullrank $J$, the pseudoinverse can be expressed as $J^{+}=$ $J^{T}\left(J J^{T}\right)^{-1}$.

In this work a general class of tasks characterized by sequences of point-to-point moves is considered. The commanded end-effector velocity is simply straight line motion towards the desired task position $\mathrm{x}_{d}$ :

$$
\dot{\mathrm{x}}_{c}=K_{e}\left(\mathrm{x}_{d}-\mathrm{x}_{a}\right) .
$$

where $\mathbf{x}_{a}$ is the actual position of the end-effector, and $K_{\epsilon}$ is a constant position error gain that is adjusted when necessary to limit the commanded end-effector velocity to a maximum allowable value.

In the event of a locked-joint failure, the actual endeffector velocity in general will not be as commanded by (6). In particular, if joint $i$ fails, the actual endeffector velocity is given by

$$
\dot{\mathrm{x}}_{a}=\left({ }^{i} J G\right) K_{e}\left(\mathrm{x}_{d}-\mathrm{x}_{a}\right) .
$$

Although $\dot{\mathrm{x}}_{a}$ may not drive the end-effector directly towards the task position, it is natural to ask whether the end-effector eventually converges to it. If not, does the end-effector converge at all, and if so, to which position?

For the class of control schemes considered here, the end-effector converges, i.e., $\dot{\mathrm{x}}_{a}=0$ if and only if $\dot{\mathrm{q}}_{a}=0$; in other words, the manipulator comes to rest if and only if all of the healthy joints are commanded zero joint velocities. The further question of whether the correct end-effector position is achieved can be answered by examining the commanded velocities of the failed joints: The correct end-effector position is achieved if and only if the commanded velocities of the failed joints are also zero.

Conditions under which motion of only the failed joint(s) is commanded for an n-DOF robot are presented in the following theorem.

Theorem 1: Consider a manipulator at a nonsingular configuration, driven by a generalized inverse control

$$
\dot{\mathbf{q}}_{c}=G \dot{\mathbf{x}}_{c},
$$

where $G=W^{-1} J^{T}\left(J W^{-1} J^{T}\right)^{-1}$ for some symmetric $W>0$. Suppose the set $S$ contains the indices of the $k$ locked joints. Let $\mathbf{j}_{i}$ and $\mathbf{w}_{i}$ denote the $i$ th column of $J$ and $W$ respectively, and let $\mathcal{R}(J)$ denote the row-space of $J$.

Then we have the following two statements.

1. If for some $\alpha_{i} \in \mathbb{R}, \sum_{i \in S} \alpha_{i} \mathbf{w}_{i} \in \mathcal{R}(J)$, then

$$
\dot{\mathrm{q}}_{c}=\sum_{i \in S} \alpha_{i} \mathbf{e}_{i} \Longleftrightarrow \dot{\mathbf{x}}_{c}=\sum_{i \in S} \alpha_{i} \dot{\mathbf{j}}_{i} .
$$

2. If $\sum_{i \in S} \alpha_{i} \mathbf{w}_{i} \notin \mathcal{R}(J)$, then $\dot{\mathbf{q}}_{c}=\sum_{i \in S} \alpha_{i} \mathbf{e}_{i}$ can never hold for nonzero $\dot{\mathbf{q}}_{c}$.

Remark: The condition $\sum_{i \in S} \alpha_{i} \mathbf{w}_{i} \in \mathcal{R}(J)$ is equivalent to rank-deficiency of a post-failure weighted Jacobian: The matrix $\left(J W^{-1}\right)_{r} \in \mathbb{R}^{m \times(n-k)}$; obtained from $J W^{-1}$ by removing the columns with indices $i \in S$, is rank-deficient.

The second statement of the theorem says that if the rank-deficiency condition does not hold at a configuration. then the manipulator cannot come to rest at that configuration.

Proof: Suppose that for some $\alpha_{i} \in \mathbb{R}$, we have $\sum_{i \in S} \alpha_{i} \mathbf{w}_{i} \in \mathcal{R}(J)$. Then,

$$
\dot{\mathrm{q}}_{c}=\sum_{i \in S} \alpha_{i} \mathbf{e}_{i} \Longrightarrow \dot{\mathrm{x}}_{c}=\sum_{i \in S} \alpha_{i} \mathbf{j}_{i}
$$


can be established simply by pre-multiplying the equation on the left by $J$. Next, consider the converse

$$
\dot{\mathbf{x}}_{c}=\sum_{i \in S} \alpha_{i} \dot{\mathbf{j}}_{i} \Longrightarrow \dot{\mathbf{q}}_{c}=\sum_{i \in S} \alpha_{i} \mathbf{e}_{i}
$$

Let $N$ be a matrix whose columns span the nullspace of $J$. Then, the set of equations

$$
\left[\begin{array}{c}
J \\
N^{T} W
\end{array}\right] \dot{\mathbf{q}}_{c}=\left[\begin{array}{c}
\sum_{i \in S} \alpha_{i} \mathbf{j}_{i} \\
0
\end{array}\right]
$$

has a unique solution $\dot{\mathbf{q}}_{c}=\sum_{i \in S} \alpha_{i} \mathbf{e}_{i}$, which completes the argument.

We prove the second statement of the theorem by contradiction. Suppose that for some nonzero $\dot{\mathbf{q}}_{c}$, we have $\dot{\mathbf{q}}_{c}=\sum_{i \in S} \alpha_{i} \mathbf{e}_{i}$. Then, it follows that $\mathrm{N}^{T} W \dot{\mathbf{q}}_{c}=0$, which implies that $\sum_{i \in S} \alpha_{i} \mathbf{w}_{i} \in \mathcal{R}(J)$, a contradiction.

Theorem 1 explicitly relates the various parameters that affect the convergence of the end-effector after undetected joint failures; the joints that have failed, the configurations at which they failed, the actual position of the end-effector, the task position for the end-effector and the inverse-kinematic control employed. Conditions under which each of the following three distinct behaviors are exhibited can be inferred from the theorem:

1. The manipulator successfully converges to the task position.

2. The manipulator converges, but to a position other than the the task position.

3. The manipulator does not converge, i.e, keeps moving forever.

As we will demonstrate in Sections III and IV, each of these behaviors is typically encountered under normal operation.

The results of Theorem 1 have great practical implications for a number of applications. For example, the workspace can be analyzed for ideal locations for critical tasks where convergence can be guaranteed under various failure scenarios. In addition, one can restrict the range of joint motions in anticipation of failures to eliminate configurations that would result in undesirable convergence behavior. One can also use the results of Theorem 1 to devise control schemes (e.g., choice of weighting $W$ ) that yield satisfactory convergence behavior.

\section{An Illustration with a 2-DOF Planar Ma- NIPULATOR}

All three behaviors listed in Section II can be illustrated even for the case of the simple planar 2-DOF

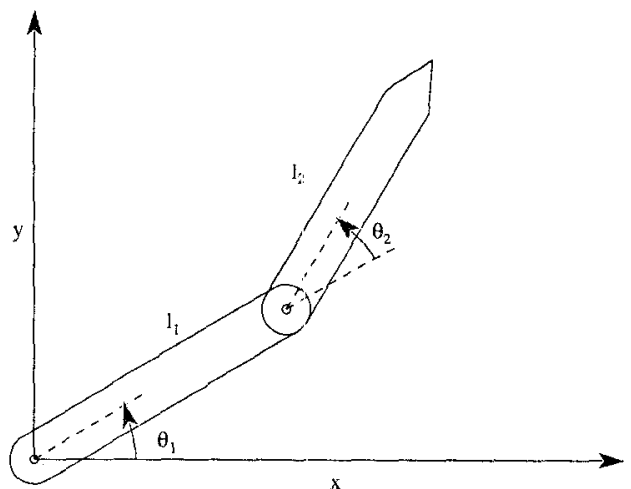

Fig. 1. A 2-DOF planar manipulator with link lengths $l_{1}=l_{2}=$ $1 \mathrm{~m}$, and joint variables $\theta_{1}$ and $\theta_{2}$.

manipulator presented in Fig. 1. This example is simple enough that we can, in addition, perform a "bruteforce" analysis of convergence. We consider the failure of the first joint at $0^{\circ}$ (without loss of generality).

Let the task position be given by $\mathbf{x}_{d}=\left[\begin{array}{ll}x_{d} & y_{d}\end{array}\right]^{T}$, where $\mathrm{x}_{d}$ is not restricted to be in the post-failure or the original workspace. When the manipulator is driven by the control input ${ }^{2}$ defined in (6), with a unit value of the positional error gain $K_{e}$, the velocity of the second joint, after a locked-joint failure of the first joint, is given by

$$
\dot{\theta}_{2}=\frac{1}{\sin \theta_{2}}\left(\left(2-x_{d}\right)\left(1+\cos \theta_{2}\right)-y_{d} \sin \theta_{2}\right) .
$$

The condition for convergence is $\dot{\theta}_{2}=0$, which results, from simple trigonometry, in

$$
\frac{x_{a}}{y_{a}}=-\frac{y_{d}}{x_{d}-2} \text {. }
$$

This condition can be reinterpreted in light of Theorem 1. From Fig. 2, we see that condition (11) is equivalent to the position vector $x_{a}$ and the commanded endeffector velocity $\dot{x}_{c}$ being orthogonal. Since $\mathbf{j}_{1}$ and $\mathbf{x}_{a}$ are also orthogonal, we conclude that $\dot{\mathbf{x}}_{c}$ and $\mathbf{j}_{1}$ must be collinear. Thus, the motion of only the first (failed) joint is commanded. This is precisely the convergence condition postulated by Theorem 1 .

It is important to point out that Theorem I only characterizes potential positions of convergence, and does not answer the questions of whether the manipulator will eventually converge to such a configuration, and if so, to which one. To address this issue, the evolution of the manipulator trajectory is investigated next for different task positions. For this analysis, it is convenient to represent $x_{d}$ and $y_{c^{\prime}}$ in polar-coordinates $(\rho, \phi)$ with origin at the second joint:

$$
x_{d}=\rho \cos (\phi)+1, \text { and } y_{d}=\rho \sin (\phi) .
$$

\footnotetext{
${ }^{2}$ Here $G$ is simply the inverse of the Jacobian, which is uniquely defined except at the singularities of the manipulator identified by the configurations where $\theta_{2}=k \pi, k$ integer.
} 


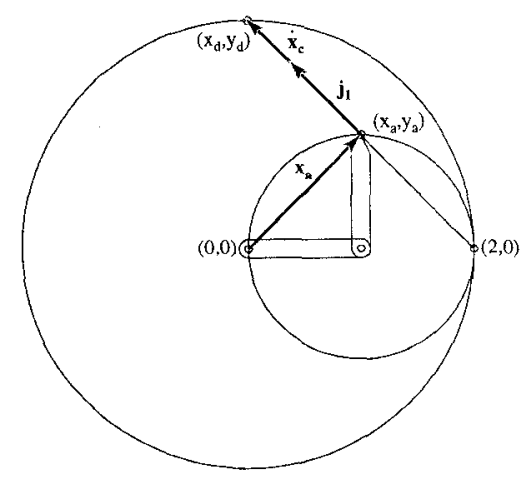

Fig. 2. Geometric interpretation of the convergence condition from Theorem 1 for the 2-DOF Example. The manipulator converges when $\dot{\mathbf{x}}_{c}$ and $\dot{j}_{1}$ are collinear. Thus, motion of only the first joint (failed) is commanded, and therefore, the manipulator does not move.

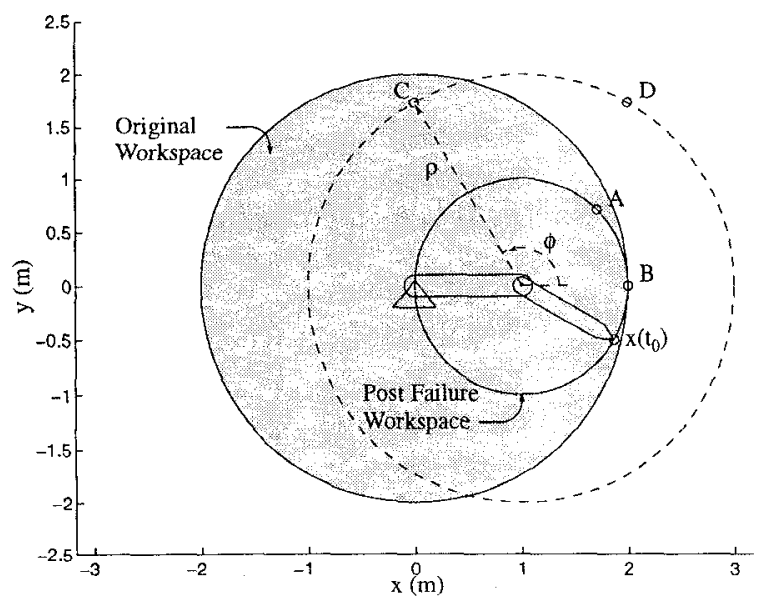

Fig. 3. A 2-DOF planar manipulator with the first joint failed at $0^{\circ}$. The shaded area represents the workspace of the healthy manipulator that reduces to the inner circle after the failure. Also shown in this plot are four task positions $\mathbf{A}, \mathbf{B}, \mathbf{C}$, and $\mathbf{D}$ for which very different convergence behaviors are observed for the same starting position $\mathbf{x}\left(t_{0}\right)$.

Four task positions that exhibit very different convergence behaviors are: $\mathbf{A}=\left(1,45^{\circ}\right), \mathbf{B}=\left(1,0^{\circ}\right)$, $\mathbf{C}=\left(2,120^{\circ}\right), \mathbf{D}=\left(2,60^{\circ}\right)$. In each case, the endeffector is initially positioned at $\mathbf{x}\left(t_{0}\right)=\left(1,-30^{\circ}\right)$, with the robot configuration given by $\theta_{1}=0^{\circ}$ and $\theta_{2}=-30^{\circ}$, and is commanded to move toward the task position until convergence. The joint-space trajectories for these cases are plotted in Figs. 4 and 5 as functions of $\theta_{2}$ and $\phi$ (since $\theta_{1}$ is always 0 ) for $\rho=1$ and $\rho=2$ respectively. For each particular task position, $\phi$ is constant and the trajectory evolves along the $\theta_{2}$ axis. For a given $\phi$, the task positions are characterized in these plots by $\theta_{2}=\phi$. Points of convergence are at the intersection of the trajectory of $\theta_{2}$ with the task position, or with the $\dot{\theta}_{2}=0$ curve $^{3}$.

Both the end-effector position error $E=\left\|\mathrm{x}_{d}-\mathrm{x}_{a}\right\|$,

${ }^{3}$ In Fig. 5 , the $\dot{\theta}_{2}=0$ curve is discontinuous at positions $\mathbf{S}_{1}$ and $\mathbf{S}_{2}$. For the given value of $\rho=2.0, \dot{\theta}_{2}$ is non-zero at these positions (from (10)).

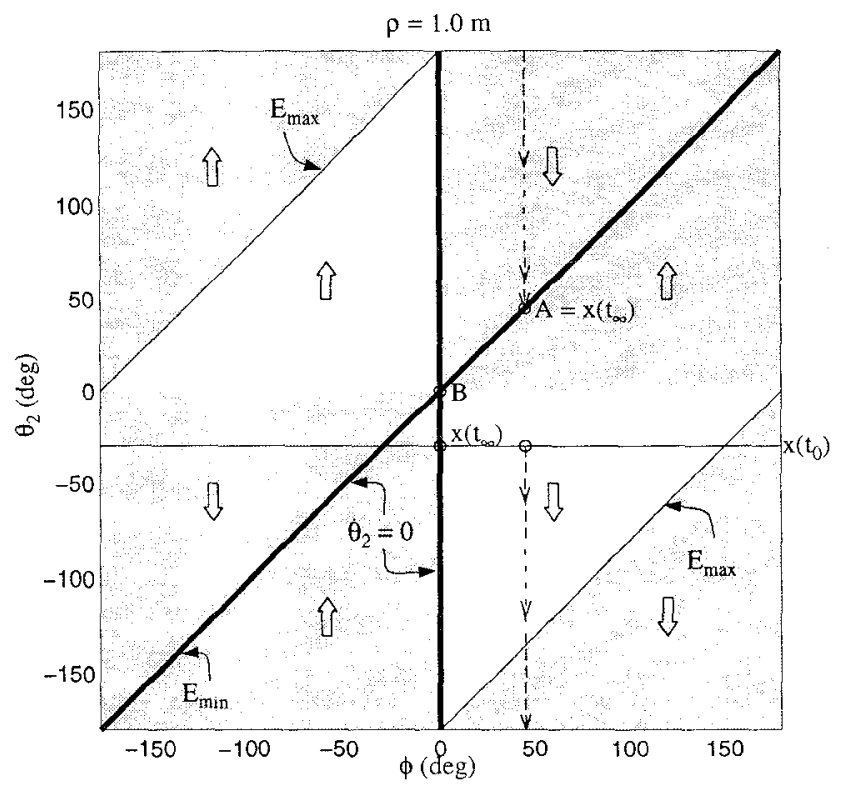

Fig. 4. Joint-space trajectories for task positions $\mathbf{A}$ and $\mathbf{B}$ Convergence to the task position is observed with $\mathbf{A}$. No motion results when the task position is $B$.

and its rate of change $\dot{E}$ are shown in the plot. The minimum and the maximum contours of the error $E$ are denoted by $E_{\min }$ and $E_{\max }$ respectively. In the shaded regions, $\dot{E}$ is negative, and it is positive in the unshaded regions. In each region, the evolution of $\theta_{2}$ is governed by (10), and is indicated in the plots by large arrows.

Case 1. Exact convergence.

From Fig. 4, it can be seen that for almost all $\phi$, the $\theta_{2}$ trajectory is drawn to the task position $\theta_{2}=\phi$. (One such example is shown with point $\mathbf{A}\left(\phi=45^{\circ}\right)$.) This behavior is exhibited only for $\rho=1$, i.e., when the task position lies in the post-failure workspace. We will see for any other value of $\rho$, not only will exact convergence not be achieved (since the task position lies outside the post-failure workspace), but also the manipulator will almost always not converge to the point closest to that desired.

Even though exact convergence occurs for $\rho=1$, the path taken by the end-effector is clearly not always the shortest. Also, the end-effector error initially increases for large regions of $\theta_{2}$ and $\phi$, leading to large excursions of the second joint, which may violate joint limit constraints and therefore prevent convergence. It is also important to note that such behavior is not preventable by simply restricting only the workspace (by limiting $\phi$ ) or only the robot configuration (by constraining $\theta_{2}$ ).

\section{Case 2. Erroneous convergence.}

We show two types of erroneous convergence. The first is the unique situation where the task position (B) is in the post-failure workspace, but the robot does not move at all (see Fig. 4). Here, $\dot{\mathbf{x}}_{c}$ and $\mathbf{j}_{1}$ are collinear 


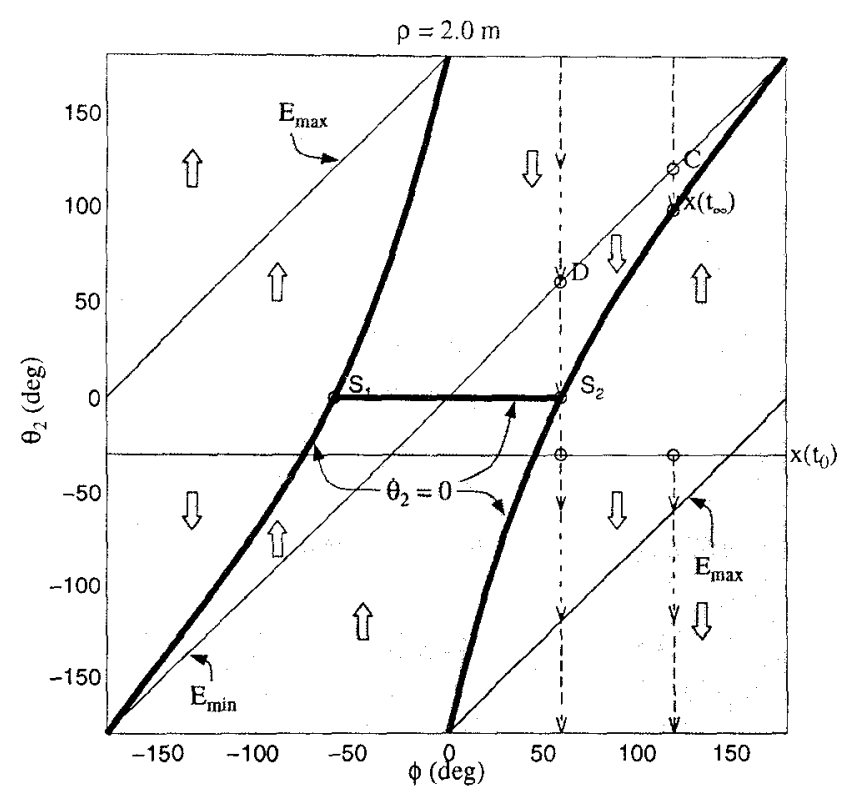

Fig. 5. Joint-space trajectories for task positions $\mathbf{C}$ and $\mathbf{D}$. The end-effector converges to a position that is not closest to $\mathbf{C}$. With $\mathbf{D}$, the manipulator does not converge at all, but cycles continuously.

and $\dot{\theta}_{2}=0$. Indeed this condition is true for any $\theta_{2}$, and therefore the robot does not move for any initial configuration. Note that this behavior will be observed at every point on the boundary of the original workspace if the first joint fails with the appropriate value for $\theta_{1}$.

The second, more common, type of erroneous convergence is observed when the task position is outside the post-failure workspace, Here, the end-effector does move, but converges to a point other than one that minimizes the error, i.e., $\theta_{2}=\phi$. One such example is the point $\mathbf{C}$ shown in Fig. 5.

Fig. 5 indicates that even for a constant value of $\rho$, very different errors may be observed at convergence depending upon the value of $\phi$. The error is a minimum for $\phi=180^{\circ}$, and rises to a maximum for $\phi$ close to $60^{\circ}$. For the special case when $\phi=60^{\circ}$, no convergence occurs; this is discussed next.

Case 3. No convergence.

$\mathbf{D}$ is an example of a task position that lies outside the original workspace, and on the line $x_{d}=2$. For such points, (10) gives $\dot{\theta}_{2}=-y_{d}$. Thus, the manipulator rotates with a constant angular velocity.

Remarks: Joint-space plots such as those in Figs. 4 and 5 are valuable workspace layout tools. These plots can be used to identify "basins of convergence" where successful task completion can be achieved even in the presence of undetected joint failures.

\section{A 3-DOF Planar Example}

Kinematic redundancy can be used to guarantee that the task positions lie within the post-failure workspace $[3,4,5,6]$. However, potential problems with

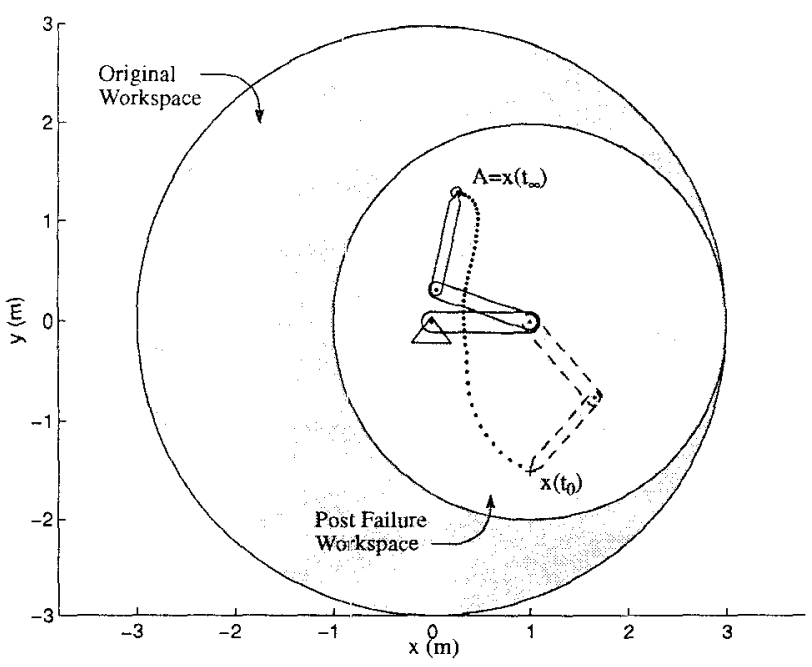

Fig. 6. Exact convergence to the task position A.

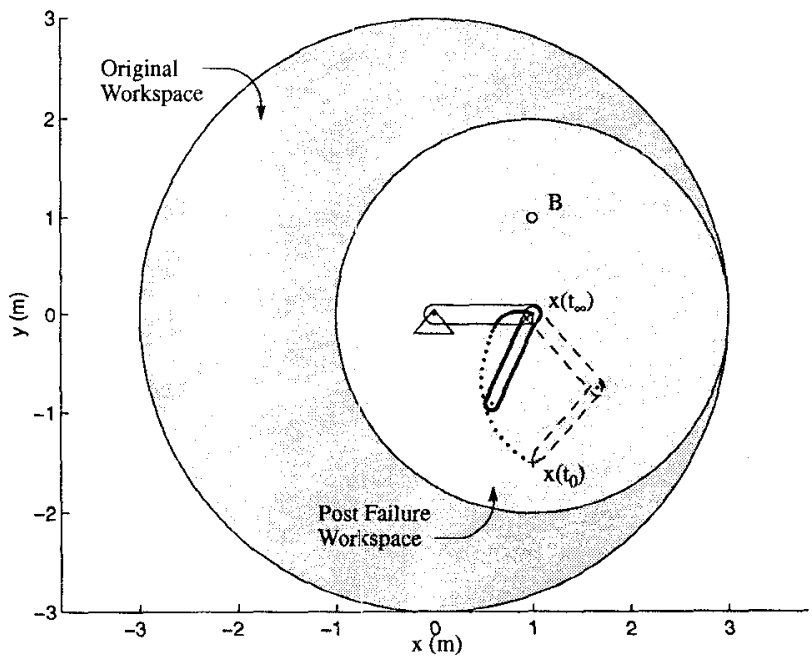

Fig. 7. Erroneous convergence when the task position $\mathbf{B}$ is in the post-failure workspace. The manipulator converges to a configuration where the "reduced-manipulator" becomes singular $\left(\theta_{3}=\pi\right)$, and $\dot{\mathbf{x}}_{\mathrm{c}}$ and $\mathbf{j}_{1}$ align.

post-failure convergence remain even in this case, as we will demonstrate with a planar 3-DOF example. Still, kinematic redundancy holds great promise for improving the convergence behavior through a judicious choice of inverse-kinematic control laws.

All the three distinct convergence behaviors are illustrated here, with four task positions $\mathbf{A}=\left(1.5,120^{\circ}\right)$, $\mathbf{B}=\left(1,90^{\circ}\right), \mathbf{C}=\left(3,131.8^{\circ}\right), \mathbf{D}=\left(3,48.2^{\circ}\right)$. Pseudoinverse control is assumed in these examples.

Fig. 6 illustrates the case when trajectory converges to the task position A. However, in Fig. 7 it is shown that erroneous convergence may result, even though the task position $\mathbf{B}$ lies in the post-failure workspace. Fig. 8 illustrates the case when the task position lies outside the post-failure workspace (but inside the original workspace), and the manipulator converges to a position other than the one that minimizes the end-effector 


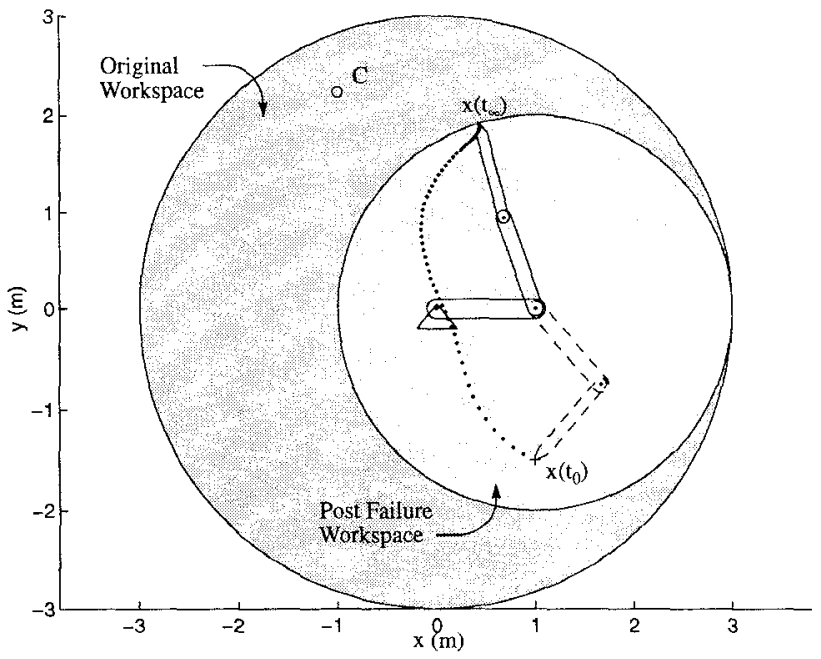

Fig. 8. Erroneous convergence when the task position $\mathbf{C}$ lies outside the post-failure workspace. Again, the manipulator converges to a configuration where the "reduced-manipulator" becomes singular $\left(\theta_{3}=0\right)$, and $\dot{\mathbf{x}}_{c}$ and $\mathbf{j}_{1}$ align.

error. Fig. 9 illustrates the case where no convergence is observed. In this case, the task point is specified to lie outside the original workspace. A potential convergence position, given by Theorem 1, coincides with a singularity of the unfailed manipulator. However, this presents no difficulty, since it is easily shown that the joint velocities commanded at the healthy joints, by the pseudoinverse, are continuous functions (this follows from $\mathbf{x}_{c}$ having no component along the singular direction). Moreover, at the singularity, the healthy joints are commanded non-zero velocities, establishing that convergence is not possible.

\section{CONCLUSION}

In this work the behavior of robotic manipulators performing point-to-point motion tasks with undetected locked-joint failures was analyzed. Conditions governing convergence issues such as whether the manipulator comes to rest, and if so, at what terminal position of the end-effector, were explicitly defined and the different possible convergence behaviors, illustrated with examples. The analysis also discussed the effect of faults on the manipulator workspace and presented valuable information for workspace layout. Our current work focuses on identifying appropriate kinematic control schemes to address the problems identified here.

\section{REFERENCES}

[1] J. F. Engelberger, "Three million hours of robot field experience," The Industrial Robot, pp. 164-168, June 1974.

[2] M. L. Visinsky, J. R. Cavallaro, and I. D. Walker, "Robotic fault detection and fault tolerance: A survey," Reliability Eng. and Sys. Safety, vol. 46, pp. 139-158, 1994.

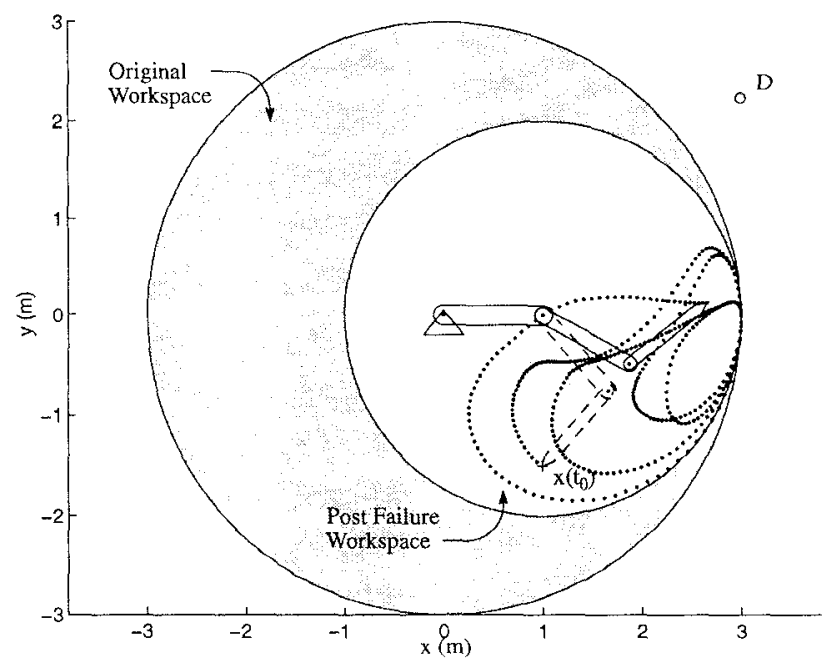

Fig. 9. No convergence. With the choice of inverse-kinematics used, nonzero joint velocities are commanded in the healthy joints everywhere, therefore the manipulator does not converge.

[3] A. A. Maciejewski, "Fault tolerant properties of kinematically redundant manipulators," in Proc. 1990 Int. Conf. on Robot. and Auto., pp. 638-642, (Cincinnati, OH), May 13-18 1990.

[4] C. L. Lewis and A. A. Maciejewski, "Dexterity optimization of kinematically redundant manipulators in the presence of failures," Comp. and Elec. Eng., vol. 20, no. 3, pp. 273-288, May 1994.

[5] C. J. J. Paredis, W. K. F. Au, and P. K. Khosla, "Kinematic design of fault tolerant manipulators," Comp. and Elec. Eng., vol. 20, no. 3, pp. 211-220, May 1994.

[6] R. G. Roberts and A. A. Maciejewski, "A local measure of fault tolerance for kinematically redundant manipulators," IEEE Trans. on Robot. and Auto., vol, 12, no. 4, pp. 543553, August 1996.

[7] J. T. Chladek, "Fault tolerance for space based manipulator mechanisms and control systems," in Proc. ISMCR '90, First Int. Symp. on Meas. and Contr. in Robot., (Houston, Texas), June 20-22 1990.

[8] E. Wu, M. Diftler, J. Hwang, and J. Chladek, "A fault tolerant joint drive system for the space shuttle remote manipulator system," in Proc. 1991 Int. Conf. on Robot. and Auta., pp. 2504-2509, (Sacramento, CA), April 9-11 1991.

[9] M. L. Visinsky, J. R. Cavallaro, and I. D. Walker, "Expert system framemwork for fault detection and fault tolerance in robotics," Comp. and Elec. Eng., vol. 20, no. 5, pp. 421-435, September 1994.

[10] M. L. Visinsky, J. R. Cavallaro, and I. D. Walker, "A dynamic fault tolerance framework for remote robots," IEEE Trans. on Rob. and Auto., vol. 11, no. 4, pp. 477-490, August 1995 .

[11] Y. Ting, S. Tosunoglu, and D. Tesar, "A control structure for fault-tolerant operation of robotic manipulators," in Proc. 1993 Int. Conf. on Rob. and Auto., pp. 684-690, (Atlanta, Georgia), May 2-6 1993.

[12] J. V. Draper, S. Handel, and C. C. Hood, "The impact of partial joint failure on teleoperation task performance," in M. Jamshidi and P. J. Eicker, editors, Robot. and Rem. Sys.: Prac, of the Fourth ANS Topic. Meet. on Robot. and Rem. Sys., pp. 433-439, (Albuquerque, New Mexico), February 24-28, 1991. 